\title{
Dynamics of Whole Genome Instability in Hypermethylated Phenotypes in Oncogenesis as DNA Oscillatory De-Methylation and Methylation
}

\author{
Lawrence M Agius* \\ Department of pathology, University of Malta Medical School, Malta
}

Submission: August 10, 2018; Published: September 26, 2018

"Correspondence Address: Lawrence M Agius, Department of pathology, University of Malta Medical School, Mater Dei Hospital Msida, TAL- QROQQ Malta, Europe, Tel: 356-21451752; Email: lawrence.agius@um.edu.mt

\begin{abstract}
Direct attribution of gene expression profiles allows for the emergence of epigenetic control of gene expression as a virtual characterization of dynamic profile changes in genomic expression and stability. As such, inclusive dynamics propose the hypomethylation status of the whole genome as expressive evidence for hypermethylation phenotypes of multiple, several sets of genes that promote and further enhance the instability of the genome within oncogenic replicative phases of cellular activity. Oncogenesis hence is a proposed system for promotion of gene regulatory restriction on the one hand and a patterned increment for further gene oscillatory pathways of promotional intent and sustainment of malignant transformation and progression.
\end{abstract}

keywords: Methylation; De-methylation; Carcinogenesis; Oscillatory balance

\section{Introduction}

DNA methylation is a fundamental process of restricted transcriptional capability borne out by promoter methylation of genes. Certain epigenetic regulators crosstalk with critical biologic pathways, such as androgen receptor singling in prostate cancer, and cooperation dynamically controls canceroriented transcriptional profiles [1]. In this regard, the overall dynamics that constitute the dimensions of transcription include developmental processes with the additional implications in oncogenesis. Ectopic epigenetic mechanisms play important roles in facilitating tumorigenesis [2]. The overall integrity of the cell methylome is further implicated in terms of sufficient import to the overall promotion or inhibition of gene expression. Gene silencing through DNA methylation at $\mathrm{CpG}$ loci around for example transcription start or enhancer sites is a major mechanism in cancer development [3]. The CpG islands are depleted during evolutionary course in a manner that may link DNA methylation with histone and chromatin epigenetics. Selective pressures might relate with specific functional impacts during carcinogenesis and drive the susceptibility of promoter regions to somatic alteration [4]. The overall dynamics are therefore involved within the system profile of turnover in which the methyl group is attached to the 5' end of the gene in proportional contributing role in DNA regulation in transcription. Host-cell DNA methylation patterns in cervical CIN2 and CIN3 are heterogeneous, with a subset displaying a cancer-like methylation-high pattern, suggestive for a higher cancer risk [5].

\section{Cell Replication}

Methylation acquires conceptual significance in terms of methylation implicating active cell replication. COX-2 promoter methylation is significantly higher in tumor tissues and is an early event for gastric carcinogenesis, implicating an initial development of this tumor type [6]. In this sense, the ongoing dynamics for gene expression is further regulated as systems of acetylation and methylation of histones. The process of evolutionary dynamics is integral to the regulatory restriction of gene expression. Aberrant small GTPase signaling is associated with a wide spectrum of human diseases including cancer [7]. The mechanistic control of active DNA demethylation is further contributory evidence for an involved course in the creation, paradoxically, of CpG Island methylation series of patterns that further constitute dimensions of repression of suppressor tumor genes in particular. A number of studies have reported the various types of cancer arising from epigenetic alteration, including epigenetic alterations occurring as a result of radiation exposure or infection [8]. Proposed increment in evolutionary control is linked intrinsically to an overall hypomethylation as borne out by dimensions of dynamic equilibrium between methylation and de-methylation. Nearly half of the human genome is comprised of repetitive elements that are highly regulated to protect the host genome from inappropriate activation, and cancer cells mis-express these elements, in part, due to decreases in DNA methylation [9]. 
In due course, the proposal for regulated gene expression is epigenetically equivalent to the proposed methylation-specific restriction enzymatic action in proposed methylation phenotype. Altered Long Interspersed Element-1 methylation in peripheral blood mononuclear cells has been simultaneously active to breast carcinogenesis due to its secretion [10]. In such instances, the overall involvement of $\mathrm{CpG}$ islands is considerably expanded within systems of methyltransferase activity. In substance, the proposed benefits for restricted gene expression contrast with active demethylation in proposed oncogenic pathways. Genomic investigations reveal novel evidence indicating that genetic predisposition and inherent drug response are critical factors for development of cancer and for poor response to therapy [11].

\section{Whole Genome Integrity}

The significance of whole genome methylation patterns account for an attempt at equilibration that is further proposed within systems of a methylome phenotype that proceeds in the context of hypomethylation in mammalian species. DNA methylation is the most extensively studied dysregulated epigenetic mechanism in colorectal carcinoma and may constitute potential blood-based biomarkers for early detection of this neoplasm [12]. Integral development allows for the significant propositions as further characterization of $\mathrm{CpG}$ island methylated phenotype, as well-evidenced in colon carcinoma, but also in gliomas and breast cancer.

Dual participation in epigenetic modification accounts for the emergence of methylation and de-methylation as productive finalization of the methylome profile. Telomerase reverse transcriptase regulates DNmethyltransferase 3B expression/ aberrant DNA methylation phenotype and AKT activation in hepatocellular carcinoma [13].

\section{Inherent Methylation}

The inherent tendencies to methylation of all forms of DNA sequence include not only genes, but also inter-genic sequences and repeat sequences. In such manner, dynamics of gene expression are further compounded by histone acetylation, methylation, phosphorylation and ubiquitination. Furthermore, the system profile coordinates introduce the mechanistic series of events in creation of restricted gene expression in methylome evolution. Methylation-associated SOX family genes have proved to be involved in multiple essential processes during carcinogenesis and are potential biomarkers for cancer diagnosis, staging, prediction or prognosis, and monitoring of response to therapy [14].

Heavy methylation of Alu and transposon repeats give further significance to the genome-wide methylation patterns in gene expression restriction and include the dynamics of turnover, particularly in terms of poorly understood active de-methylation. DNMT3A and TET1 cooperate to regulate promoter epigenetic landscapes in mouse embryonic stem cells [15].
In view of onset and of progression of such regulatory circuits, the proposed methylome patterns allow for $\mathrm{CpG}$ islands to oscillate within various change modifications of the methylome phenotypes. As a non-mutagenic human carcinogen, arsenic's carcinogenic activity is likely the result of epigenetic changes, particularly alterations in DNA methylation [16]. Included dimensions of methylomes of cell DNA propose the inclusion for further modification that relate particularly and in often specific manner to cell replicative activity. DNA methylation accumulation is associated with molecular irreversibleness and gastric carcinogenesis after H. pylori eradication [17].

\section{Segregation}

Functional segregation of chromatin is also an important mechanism in the promotion of epigenetic regulation, as further illustrated by the general concept of segregation of DNA and other system methylome dynamics that include systems of inducible modification in gene expression. Arsenic exposure causes dynamic changes in histone acetylation and methylation patterns during arsenic-induced cancer development [18].

The concept of epigenetic landscapes is hence integral to the promotional dimensions of regulatory mechanistic steps involving the whole integrative functionality of the genomewide pathways. Ionising radiation can target the cellular epigenome also; they consist of specific covalent modifications of chromatin components such as methylation of DNA, histone modifications, and control performed by non-coding RNAs [19]. The propositional inclusion of substantial integration of such systems allow for the inclusion for further methylation phenotype patterns as applicable to systems of progression of oncogenesis and spread. The performance attributes of methylome patterns hence is especially motivated within the active phases of cell replication as proposed by systems for induced gene expression modification.

\section{De-Methylation}

Proposed active de-methylation is conceptualized as system promotion of enzymatic removal of methyl groups and as nucleotide-excision repair. Embodied dimensions for increased turnover include the system profile determinations in correlative systems for control in methylome dynamics. The inclusion for further modification is projected compromise of system pathways that epigenetically regulate whole genome shifts in integral gene expression. High-throughput screening with nucleosome substrate identifies small-molecule inhibitors of the human histone lysine methyltransferase NSD2 [20].

Hypermethylation phenotypes involve several genes within a given genome and allow for the emergence of aberrant promoter methylation patterns. Also, DNA methylation works in concert with histone modifications in DNA regulatory patterns in gene expression. Once implicated in status identification of an otherwise hypomethylated genome, it is also involved in the histone modifications of epigenetic information. The lysine- 


\section{Cancer Therapy \& Oncology International Journal}

specific histone demethylase $1 \mathrm{~A}$ was the first demethylase to challenge the concept of the irreversible nature of methylation marks and is implicated in carcinogenesis and epigenetic development regulation; it interacts with transcription factors, promoters, enhancers, oncoproteins, and tumor-associated genes [21].

\section{Endoparasitic Sequences}

Activation of endoparasitic sequences may result in aneuploidy and also involve the hypomethylation status of the whole genome in the activation of various genes that express such intermediate metabolites as growth-promoters. Genome instability allows for the emergence expression profiles of hypermethylated promoters as well exemplified by predominantly suppressor tumor genes. Differential variability in cancer contributes to cancer heterogeneity and is crucial in detecting epigenetic field defects, DNA methylation alterations happening early in carcinogenesis [22].

Hypermethylation phenotypes are an expected revolutionary system profile within the system identification of pathway formulation involving multiple genes within the specifics of a whole integral genome. DNA methylation patterns specify cell-type identity during cellular differentiation and therefore provide opportunities for the molecular analysis of tumors [23]. Individual tumors share multiple sequence methylation patterns, but also exhibit unique traits of hypermethylation as proposed also in gliomas, colon carcinoma and breast cancer. The evolutionary dynamics incorporate a genomic instability that further compromises gene expression patterns throughout the whole genome. Telomere-related genes that are methylated may be a mechanism through which blood leukocyte telomere length dynamics reflect cancer risk [24].

\section{Conclusion}

The turnover for the reproducibility dynamics of restricted gene expression in hypermethylation phenotypes is exemplary evidence in the production and sustained reproduction of events for oncogenesis as further proposed by systems such as endoparasitic sequence replication. Transposons are further elaborated sequences in the mechanistic pathways of induced change in genome-wide re-characterization of gene expression as further proposed by such pathways as hypermethylation phenotypes.

Performance increments as evidenced in an overall genome hypomethylation series of changes include the participation of events of promotional intent and consequence. The performance of restricted gene expression oscillates as systems of otherwise dynamic gene hypomethylation within the whole genome. Cooperative genomic and epigenomic sequence methylation is inclusive characterization of events that signify the turnover essentials in participation of gene expression based especially on the overall systems of histone acetylation and methylation and as sequestration inclusion phenomena of such turnover of genomic and epigenetic control of gene expression.

\section{References}

1. Liao Y, Xu K (2018) Epigenetic regulatorn of prostate cancer: the theories and the clinical implications. Asian J Androl.

2. Feng J, Zhang Y, She X, Sun Y, Fan L, et al. (2018) Hypermethylated gene ANKDD1A is a candidate tumor suppressor that interacts with FIH1 and decreases HIF1alpha stability to inhibit cell autophagy in the glioblastoma multiform hypoxia microenvironment. Oncogene.

3. Kordowski F, Kolarova J, Schafmayer C, Buch S, Goldmann T, et al. (2018) Aberrant DNA methylation of ADAMTS16 in colorectal and other epithelial cancers. BMC Cancer 18(1): 796.

4. Thompson JA, Christensen BC, Marsit CJ (2018) Pan-cancer analysis reveals differential susceptibility of bidirectional gene promoters to DNA methylation, somatic mutations, and copy number alterations. Int J Mol Sci 19(8).

5. Verlaat W, van Leeuwen RW, Noviarti PW, Schuuring E, Meijer CJLM, et al. (2018) Host-cell DNA methylation patterns during high-risk HPVinduced carcinogenesis reveal a heterogeneous nature of cervical precancer. Epigenetics 24:1-10.

6. Kim HG, Ryu SY, Lee KH, Lee JH, Kim DY (2018) Quantitative analysis of COX-2 promoter methylation in gastric carcinoma. Ann Surg Treat Res 95(2): 55-63.

7. Huang M, Qi TF, Li L, Zhang G, Wang Y (2018) A targeted quantitative proteomic approach assesses the reprogramming of small GTPases during melanoma metastasis. Cancer Res 17-3811.

8. Bae JM (2018) Researches of epigenetic epidemiology for infections and radiation as carcinogen" J Prev Med Public Health 51(4): 169-172.

9. Ishak CA, Classon M, De Carvalho DD (2018) Deregulation of retroelements as an emerging therapeutic opportunity in cancer. Trends Cancer 4(8): 583-597.

10. Arayataweegool A, Srisuttee R, Mahattanasakul P, Tangiaturonsasme N, Kerekhanjanarong V, et al. (2018) Head and neck squamous cell carcinoma drives line-1 hypomethylation in the peripheral blood mononuclear cells. Oral Dis.

11. Kaina B, Izzotti A, Xu J, Christmann M, Pulliero A, et al. (2018) Inherent and toxicant-provoked reduction in DNA repair capacity: a key mechanism for personalised risk assessment, cancer prevention and intervention, and response to therapy" Int J Hyg Environ Health 221(7): 993-2006.

12. Dong L, Ren H (2018) Blood-based DNA methylation biomarkers for early detection of colorectal cancer. J Proteomics Bioinform 11(6): 120-126.

i. Yu J, Yuan X, Sjoholm L, Liu T, Kong F, et al. (2018) Telomerase reverse transcriptase regulates DNMT3B expression/aberrant DNA methylation phenotype and AKT activation in hepatocellular carcinoma. Cancer Lett 434: 33-41.

13. Zhou JD, Wang YX, Zhang TJ, Li XX, Gu Y, et al. (2018) Identification and validation of SRY-box containing gene family member SOX30 methylation as a prognostic and predictive biomarker in myeloid malignancies" Clin Epigenetics 10: 92.

14.Gu T, Lin X, Cullen SM, Luo M, Jeong M, et al. (2018) DNMT3A and TET1 cooperate to regulate promoter epigenetic landscapes in mouse embryonic stem cells. Genome Biol 19(1): 88.

15. Guo X, Chen X, Wang J, Liu Z, Gaile D et al. (2018) Multi-generational impacts of arsenic exposure on genome-wide DNA methylation and the implications for arsenic-induced skin lesions. Environ Int 119: 250-263.

16. Sahara S, Tahara T, Horiguchi N, Kato T, Shinkai Y, et al. (2018) DNA methylation accumulation in gastric mucosa adjacent to cancer after Helicobacter pylori eradication. Int J Cancer. 


\section{Cancer Therapy \& Oncology International Journal}

17. Ge Y, Zhu J, Wang X, Zheng N, Tu C, et al. (2018) Mapping dynamic histone modification patterns during arsenic-induced malignant transformation of human bladder cells. Toxic Appl Pharmacy 355: 164-173.

18. Minuses IR, Ewing LE, Kutanzi KR, Griffin RJ, Koturbash I (2018) DNA methylation in radiation-induced carcinogenesis: experimental evidence and clinical perspectives. Crit Rev Oncog 23(1-2): 1-11.

19. Coussens NP, Kales SC, Henderson MJ, Lee OW, Horiuchi KY, et al (2018) High-throughput screening with nucleosome substrate identifies small-molecule inhibitors of the human histone lysine methyltransferase NSD2. J Biol Chem.

20. Ismail T, Lee HK, Kim C, Kwon T, Park TJ, et al. (2018) KDM1A microenvironment, its oncogenic potential, and therapeutic significance. Epigenetics Chromatin 11(1): 33
21. Wang Y, Teschendorff AE, Widschwendter M, Wang S (2017) Accounting for differential variability in detecting differentially methylated regions. Brief Bioinform.

22. Bormann F, Rodriguez-Paredes M, Lasitschka F, Edelmann D, Musch T, et al. (2018) Cell-of-Origin DNA methylation signatures are maintained during colorectal carcinogenesis. Cell Rep 23(11): 3407-3418.

23. Joyce BT, Zheng Y, Nannini D, Zhang Z, Liu L, et al. (2018) DNA methylation of telomere-related genes and cancer risk" Cancer Prev Res (Phila) 11(8): 511-522

\section{Your next submission with Juniper Publishers will reach you the below assets}

- Quality Editorial service

- Swift Peer Review

- Reprints availability

- E-prints Service

- Manuscript Podcast for convenient understanding

- Global attainment for your research

- Manuscript accessibility in different formats ( Pdf, E-pub, Full Text, Audio)

- Unceasing customer service

Track the below URL for one-step submission https://juniperpublishers.com/online-submission.php 